\title{
Effects of rumen-protected creatine pyruvate on blood biochemical parameters and rumen fluid characteristics in transported beef cattle
}

\author{
Kang Mao ${ }^{1}$, Guwei Lu', Yanjiao Li ${ }^{2}$, Yitian Zang ${ }^{3}$, Xianghui Zhao², Qinghua Qiu ${ }^{2}$, Mingren Qu ${ }^{1}$ and
} Kehui Ouyang ${ }^{2}$

\begin{abstract}
Background: The fasting and stress associated with road transportation contributes to a lack of energy and a decline in the immune system of beef cattle. Therefore, it is essential for beef cattle to enhance energy reserves before transportation. Creatine pyruvate ( $\mathrm{CrPyr}$ ) is a new multifunctional nutrient that can provide both pyruvate and creatine, which are two intermediate products of energy metabolism. To investigate the effects of transport and rumen-protected (RP)-CrPyr on the blood biochemical parameters and rumen fluid characteristics of beef cattle, twenty male Simmental crossbred cattle $(659 \pm 16 \mathrm{~kg})$ aged 18 months were randomly allocated to four groups $(n=5)$ using a $2 \times 2$ factorial arrangement with two RP-CrPyr supplemental levels ( 0 or $140 \mathrm{~g} / \mathrm{d}$ ) and two transport treatments ( 5 min or 12 h):T_CrPyr140,T_CrPyrO, NT_CrPyr140, and NT_CrPyr0. After feeding for 30 days, three cattle per treatment were slaughtered.

Results: Compared with nontransport, transport decreased the total antioxidant capacity, catalase activity, contents of IgA, interferon $\gamma$, interleukin-1 $\beta$ (IL-1 $\beta$ ), and IL-6 in serum, and the amounts of total volatile fatty acids (TVFA), acetate, and butyrate in rumen $(P<0.05)$; increased the serum lipopolysaccharide (LPS) level, contents of rumen LPS and ammonia nitrogen $(P<0.05)$. RP-CrPyr supplementation decreased the levels of cortisol and LPS in serum and the butyrate concentration in the rumen of beef cattle compared with those in the unsupplemented groups $(P<0.05)$. RP-CrPyr and transport interaction had a significant effect on the contents of serum tumour necrosis factor-a, IL-6, LPS, ruminal $\mathrm{pH}$, acetate content, and acetate/propionate $(P<0.05)$. In terms of ruminal bacterial composition, group $T_{-}$ CrPyr0 increased the Prevotella genus abundance compared with group NT_CrPyrO $(P<0.05)$, while group T_CrPyr140 increased Firmicutes phylum abundance and decreased Bacteroidetes phylum and genus Prevotella abundance compared with group T_CrPyrO $(P<0.05)$. Moreover, Bacteroidetes was positively correlated with serum LPS.
\end{abstract}

Conclusions: These results indicated that dietary supplementation with RP-CrPyr might be beneficial to alleviate transport stress by decreasing serum cortisol and LPS levels and promoting the restoration of the rumen natural flora.

Keywords: Antioxidant activity, Beef cattle, Creatine pyruvate, Rumen fermentation transport stress

*Correspondence: yanjiaoli221@163.com

2 Jiangxi Province Key Laboratory of Animal Nutrition/Animal Nutrition and Feed Safety Innovation Team, College of Animal Science and Technology, Jiangxi Agricultural University, Nanchang, China Full list of author information is available at the end of the article

\section{Background}

In China, allochthonous fattening is the dominant beef cattle production model. The north reproduction and south raise have become inherent patterns. The fasting 
and stress associated with long-distance transportation contributes to a lack of energy and a decline in the immune system of beef cattle. Subsequently, transport stress syndrome of beef cattle (TSSBC) with significant morbidity and high mortality rates frequently occurs following long-distance transportation and leads to enormous economic losses [1, 2]. Ruminal microecological balance is essential for adequate nutrient supply and immunity improvement. Microbial fermentation of feedstuff in the rumen produces volatile fatty acids (VFAs) and microbial protein to provide the bulk of the energy and protein required by the host animal [3]. However, several studies show that transport stress affects ruminal microbiota abundance and diversity $[4,5]$, which might further influence host feed digestion and metabolism. Moreover, animals exposed to stress emit gastrointestinal pathogens that can increase the secretion of virulence factors [6]. Thus, it is essential for beef cattle to enhance energy reserves before transportation and maintain the ruminal microecological balance and function during transportation to improve ruminant health.

Creatine pyruvate (CrPyr), with a molecular formula of $\mathrm{C}_{7} \mathrm{H}_{13} \mathrm{~N}_{3} \mathrm{O}_{5}$ and a molecular weight of 219.20 , is a new multifunctional nutrient that contains pyruvate and creatine at a ratio of 40:60 and can provide both pyruvate and creatine [7]. For humans and nonruminants, several studies have shown that CrPyr can enhance body energy metabolism. Jäger et al. [8] reported that $\mathrm{CrPyr}$ could promote aerobic metabolism in athletes to enhance endurance performance. Chen [9] found that CrPyr supplementation increased the creatine kinase activity and phosphocreatine concentration in the muscle of broilers and increased glycogen reserve in muscle by reducing the decomposition of glycogen by decreasing phosphorylaseb activity. Recently, it was reported that in ovo feeding of $\mathrm{CrPyr}$ enhanced energy reserves in the liver and muscle of broilers on the day of hatching, increased glycolytic enzyme activity and promoted glycolysis in the chest [10]. In addition, our previous study showed that nonbypassed CrPyr could relieve the heat stress of beef cattle by improving antioxidant activity and rumen microbial protein synthesis [11]. Given these properties of CrPyr, we investigated the influences of RP-CrPyr (rumen bypass ratio is $82.8 \%$ ) on serum hormone levels, antioxidant activity, immunity, rumen fermentation, and the ruminal microorganism community of cattle with transport treatment. We hypothesized that the present work will lay a foundation for the further study of RP-CrPyr supplementation in the transport stress of cattle. Therefore, this study was designed to test this hypothesis.

\section{Results}

\section{Serum hormones and antioxidant parameters}

The results of serum hormones and antioxidant parameters are presented in Table 1. Compared with the nontransport treatment, transport significantly decreased the serum T-AOC $(1.98$ vs. $2.03 \mathrm{mmol} / \mathrm{L} ; P<0.01)$ and CAT activity $(28.41$ vs. $40.53 \mathrm{U} / \mathrm{mL} ; P<0.05)$ in beef cattle. Supplementation with RP-CrPyr significantly decreased the concentrations of serum cortisol (62.63 vs. $80.58 \mathrm{ng} /$ $\mathrm{mL} ; P<0.05)$ and tended to decrease the concentrations of serum T3 (1.10 vs. $1.18 \mathrm{ng} / \mathrm{mL} ; P=0.081)$ and MDA

Table 1 Effects of transport and rumen-protected creatine pyruvate on the serum hormones and antioxidant parameters in the beef cattle

\begin{tabular}{|c|c|c|c|c|c|c|c|c|}
\hline \multirow[t]{3}{*}{ Item } & \multicolumn{4}{|l|}{ Treatment $^{a}$} & \multirow[t]{3}{*}{ SEM } & \multirow{2}{*}{\multicolumn{3}{|c|}{$P$-value }} \\
\hline & \multicolumn{2}{|l|}{ Transport } & \multicolumn{2}{|c|}{ Nontransport } & & & & \\
\hline & RP-CrPyr 140 & RP-CrPyr 0 & RP-CrPyr 140 & RP-CrPyr 0 & & Transport & RP-CrPyr & $\begin{array}{l}\text { Transport } \\
\times \\
\text { RP-CrPyr }\end{array}$ \\
\hline T3 (ng/mL) & 1.12 & 1.22 & 1.07 & 1.14 & 0.031 & 0.151 & 0.081 & 0.732 \\
\hline $\mathrm{T} 4$ (ng/mL) & 74.65 & 72.16 & 68.39 & 76.90 & 5.682 & 0.815 & 0.365 & 0.117 \\
\hline Cortisol (ng/mL) & 62.99 & 84.66 & 62.27 & 76.49 & 12.620 & 0.430 & 0.010 & 0.505 \\
\hline MDA (nmol/mL) & 5.61 & 7.92 & 4.19 & 6.07 & 0.539 & 0.167 & 0.088 & 0.853 \\
\hline $\mathrm{SOD}(\mathrm{U} / \mathrm{mL})$ & 88.77 & 80.15 & 85.60 & 84.41 & 2.165 & 0.908 & 0.317 & 0.442 \\
\hline T-AOC (mmol/L) & 2.00 & 1.98 & 2.04 & 2.02 & 0.005 & 0.002 & 0.117 & 0.882 \\
\hline GSH-Px (U/mL) & 109.65 & 125.14 & 133.14 & 136.24 & 6.864 & 0.174 & 0.607 & 0.445 \\
\hline CAT $(\mathrm{U} / \mathrm{mL})$ & 27.15 & 24.50 & 41.36 & 39.68 & 3.253 & 0.010 & 0.634 & 0.914 \\
\hline
\end{tabular}

${ }^{a}$ A $2 \times 2$ factorial arrangement with 2 supplemental levels of rumen-protected creatine pyruvate (RP-CrPyr) in basal diets ( 0 or $140 \mathrm{~g} / \mathrm{d}$ for each cattle) and 2 transport treatment (transport 0 or $12 \mathrm{~h}$ before slaughter)

b Transport $\times$ RP-CrPyr, the interaction between Transport and RP-CrPyr

SEM Stand error of mean, T3 Triiodothyronine, T4 Thyroxine, MDA Malondialdehyde, SOD Superoxide dismutase, T-AOC Total antioxidant capacity, GSH-Px Glutathione per-oxidase, CAT Catalase 
( 4.90 vs. $6.99 \mathrm{nmol} / \mathrm{mL} ; P=0.088$ ) compared with cattle given $0 \mathrm{~g} /$ day RP-CrPyr treatment. There was no interaction on serum antioxidant parameters.

\section{Immunoglobulin, inflammatory factor, lipopolysaccharide,} and extracellular DNA levels

As shown in Table 2, compared with the nontransport treatment, the contents of serum IgA $(0.35$ vs. $0.45 \mathrm{~g} / \mathrm{L})$, IFN- $\gamma$ ( 32.69 vs. $44.54 \mathrm{pg} / \mathrm{mL}$ ), IL- $1 \beta$ ( 20.87 vs. $29.86 \mathrm{pg} /$ $\mathrm{mL})$, and IL-6 (100.37 vs. $138.97 \mathrm{pg} / \mathrm{mL})$ were significantly decreased $(P<0.05)$, and the levels of ruminal LPS $(2.30$ vs. $0.76 \mathrm{EU} / \mathrm{mL} ; P<0.001)$ and serum LPS $(22.94$ vs. $10.98 \mathrm{EU} / \mathrm{mL} ; P<0.01)$ were significantly increased in beef cattle in the transport treatment. However, transport treatment did not significantly affect the contents of IgG, IgM, and TNF- $\alpha$ in serum. Moreover, there was no significant difference in the levels of immunoglobulin and inflammatory factors between the RP-CrPyr-supplemented and unsupplemented groups $(P>0.05)$. However, dietary RP-CrPyr supplementation decreased the serum LPS level $(13.85$ vs. $20.07 \mathrm{EU} / \mathrm{mL} ; P<0.05)$ of beef cattle than those in the unsupplemented groups. Transport or RP-CrPyr did not affect the contents of eDNA in serum or rumen fluid $(P>0.05)$. RP-CrPyr and transport interaction had a significant effect on serum TNF- $\alpha$ and IL-6 $(P<0.05)$.

\section{Rumen fermentation characteristics}

The results of rumen fermentation characteristics are presented in Table 3. Compared with the nontransport treatment, transport tended to increase the rumen fluid $\mathrm{pH}(6.80$ vs. $6.51 ; P=0.087)$ and decrease ruminal propionate content ( 11.25 vs. $12.61 \mathrm{mmol} / \mathrm{L} ; P=0.070)$; significantly increased the ruminal $\mathrm{NH}_{3}-\mathrm{N}$ content (18.78 vs. $8.22 \mathrm{mg} / \mathrm{dL} ; P<0.001)$, decreased the concentrations of ruminal TVFA (76.73 vs. $86.49 \mathrm{mmol} / \mathrm{L} ; P<0.01$ ), acetate $(58.36$ vs. $64.26 \mathrm{mmol} / \mathrm{L} ; P<0.05)$ and butyrate (7.11 vs. $9.92 \mathrm{mmol} / \mathrm{L} ; P<0.001)$. Supplementation with RP-CrPyr tended to decrease the concentration of ruminal VFAs (79.12 vs. $84.10 \mathrm{mmol} / \mathrm{L} ; P=0.050)$ and significantly decrease the ruminal butyrate content $(7.44 \mathrm{vs}$. $9.59 \mathrm{mmol} / \mathrm{L} ; P<0.001)$. Transport or RP-CrPyr did not affect the content of ruminal MCP $(P>0.05)$. Except for rumen fluid $\mathrm{pH}$, ruminal acetate, acetate/propionate, and serum LPS, there was no significant RP-CrPyr and transport interaction effect on the other characteristics.

\section{Alpha-Diversity measures and OTU analysis}

The alpha diversity index is mainly used to evaluate the richness and uniformity of microorganisms in samples. As shown in Table 4, at the same sequencing depth, the alpha values of the four groups were not significantly different in Ace, Chao1, Shannon, and Simpson $(P>0.05)$.

Table 2 Effects of transport and rumen-protected creatine pyruvate on the contents of immunoglobulin and inflammatory factor in serum, lipopolysaccharide and extracellular DNA in serum and rumen fluid of beef cattle

\begin{tabular}{|c|c|c|c|c|c|c|c|c|}
\hline \multirow[t]{3}{*}{ Item } & \multicolumn{4}{|l|}{ Treatment $^{1}$} & \multirow[t]{3}{*}{ SEM } & \multirow{2}{*}{\multicolumn{3}{|c|}{$P$-value }} \\
\hline & \multicolumn{2}{|l|}{ Transport } & \multicolumn{2}{|c|}{ Nontransport } & & & & \\
\hline & $\mathrm{RP}-\mathrm{CrPyr}_{140}$ & $\mathrm{RP}-\mathrm{CrPyr}{ }_{0}$ & $\mathrm{RP}-\mathrm{CrPyr}_{140}$ & $\mathrm{RP}-\mathrm{CrPyr}_{0}$ & & Transport & RP-CrPyr & $\begin{array}{l}\text { Transport } \\
\times \\
\text { RP-CrPyr }\end{array}$ \\
\hline \multicolumn{9}{|l|}{ Serum } \\
\hline $\lg G(g / L)$ & 9.44 & 8.75 & 9.93 & 9.80 & 0.607 & 0.539 & 0.744 & 0.820 \\
\hline $\operatorname{lgM}(g / L)$ & 2.44 & 2.36 & 2.59 & 2.47 & 0.167 & 0.689 & 0.776 & 0.960 \\
\hline $\lg A(g / L)$ & 0.34 & 0.36 & 0.44 & 0.47 & 0.021 & 0.028 & 0.543 & 0.983 \\
\hline TNF-a (pg/mL) & 59.24 & 47.91 & 46.75 & 57.89 & 2.594 & 0.791 & 0.984 & 0.040 \\
\hline $\mathrm{IFN}-\gamma(\mathrm{pg} / \mathrm{mL})$ & 31.92 & 33.45 & 41.83 & 47.25 & 1.573 & 0.005 & 0.301 & 0.554 \\
\hline $\mathrm{IL}-1 \beta(\mathrm{pg} / \mathrm{mL})$ & 22.87 & 18.86 & 28.83 & 30.89 & 1.643 & 0.026 & 0.773 & 0.383 \\
\hline IL-6 (pg/mL) & $87.00^{c}$ & $113.72^{b c}$ & $149.19^{a}$ & $128.75^{\mathrm{ab}}$ & 4.301 & 0.002 & 0.725 & 0.025 \\
\hline LPS (EU/mL) & $16.71^{b}$ & $29.18^{a}$ & $11.00^{\mathrm{b}}$ & $10.96^{\mathrm{b}}$ & 2.463 & 0.001 & 0.032 & 0.031 \\
\hline eDNA (ng/uL) & 2.36 & 2.39 & 2.11 & 2.17 & 0.146 & 0.459 & 0.882 & 0.975 \\
\hline \multicolumn{9}{|l|}{ Rumen } \\
\hline LPS (EU/mL) & 2.19 & 2.41 & 0.64 & 0.89 & 0.074 & $<0.001$ & 0.143 & 0.929 \\
\hline 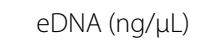 & 19.95 & 20.94 & 26.12 & 17.00 & 1.503 & 0.720 & 0.213 & 0.131 \\
\hline
\end{tabular}

$\mathrm{a}, \mathrm{b}, \mathrm{c}$ Means within a row with no common superscript differ significantly $(P<0.05)$

${ }^{1}$ A $2 \times 2$ factorial arrangement with 2 supplemental levels of rumen-protected creatine pyruvate (RP-CrPyr) in basal diets ( 0 or $140 \mathrm{~g} / \mathrm{d}$ for each cattle) and 2 transport treatment (transport 0 or $12 \mathrm{~h}$ before slaughter)

2 Transport $\times$ RP-CrPyr, the interaction between Transport and RP-CrPyr

SEM Stand error of mean, TNF- $\alpha$ Tumor necrosis factor- $a$, IFN- $\gamma$ Interferon $\gamma$, IL-1 $\beta$ Interleukin-1 $\beta$, IL-6 Interleukin-6, LPS Lipopolysaccharide, eDNA Extracellular DNA 
Table 3 Effects of transport and rumen-protected creatine pyruvate on the rumen fermentation characteristics of beef cattle

\begin{tabular}{|c|c|c|c|c|c|c|c|c|}
\hline \multirow[t]{3}{*}{ Item } & \multicolumn{4}{|l|}{ Treatment $^{1}$} & \multirow[t]{3}{*}{ SEM } & \multirow{2}{*}{\multicolumn{3}{|c|}{$P$-value }} \\
\hline & \multicolumn{2}{|l|}{ Transport } & \multicolumn{2}{|l|}{ Nontransport } & & & & \\
\hline & $\mathrm{RP}-\mathrm{CrPyr}_{140}$ & RP-CrPyr ${ }_{0}$ & $\mathrm{RP} \mathrm{CrPyr}_{140}$ & $\mathrm{RP}-\mathrm{CrPyr} \mathrm{r}_{0}$ & & Transport & RP-CrPyr & $\begin{array}{l}\text { Transport } \\
\times \\
\text { RP-CrPyr }{ }^{2}\end{array}$ \\
\hline $\mathrm{pH}$ & 7.02 & 6.58 & 6.37 & 6.65 & 0.076 & 0.087 & 0.619 & 0.046 \\
\hline $\mathrm{MCP}(\mathrm{mg} / \mathrm{mL})$ & 0.22 & 0.31 & 0.23 & 0.19 & 0.021 & 0.188 & 0.639 & 0.092 \\
\hline $\mathrm{NH}_{3}-\mathrm{N}(\mathrm{mg} / \mathrm{dL})$ & 16.64 & 20.93 & 8.18 & 8.26 & 0.672 & $<0.001$ & 0.143 & 0.155 \\
\hline TVFA (mmol/L) & 72.18 & 81.28 & 86.06 & 86.92 & 1.997 & 0.001 & 0.050 & 0.060 \\
\hline Acetate $(\mathrm{mmol} / \mathrm{L})$ & $54.75^{b}$ & $61.98^{\mathrm{a}}$ & $66.21^{a}$ & $62.32^{\mathrm{a}}$ & 5.374 & 0.026 & 0.461 & 0.033 \\
\hline Propionate (mmol/L) & 11.35 & 11.15 & 11.67 & 13.56 & 1.383 & 0.070 & 0.231 & 0.528 \\
\hline Butyrate (mmol/L) & 6.08 & 8.15 & 8.81 & 11.04 & 1.910 & $<0.001$ & $<0.001$ & 0.819 \\
\hline Acetate/Propionate & 4.89 & 5.61 & 4.60 & 5.68 & 0.201 & 0.755 & 0.618 & 0.029 \\
\hline
\end{tabular}

a,b,c Means within a row with no common superscript differ significantly $(P<0.05)$

${ }^{1}$ A $2 \times 2$ factorial arrangement with 2 supplemental levels of rumen-protected creatine pyruvate (RP-CrPyr) in basal diets ( 0 or $140 \mathrm{~g} / \mathrm{d}$ for each cattle) and 2 transport treatment (transport 0 or $12 \mathrm{~h}$ before slaughter)

2 Transport $\times$ RP-CrPyr, the interaction between Transport and RP-CrPyr

SEM Stand error of mean, MCP Microbial crude protein, $\mathrm{NH}_{3}-\mathrm{N}$ Ammonia nitrogen, TVFA Total volatile fatty acids

Table 4 Effects of transport and rumen-protected creatine pyruvate on the Alpha diversity indices

\begin{tabular}{|c|c|c|c|c|c|c|c|c|}
\hline \multirow[t]{3}{*}{ Item } & \multicolumn{4}{|l|}{ Treatment $^{\mathrm{a}}$} & \multirow[t]{3}{*}{ SEM } & \multirow{2}{*}{\multicolumn{3}{|c|}{$P$-value }} \\
\hline & \multicolumn{2}{|l|}{ Transport } & \multicolumn{2}{|c|}{ Nontransport } & & & & \\
\hline & $\mathrm{RP}-\mathrm{CrPyr}_{140}$ & $\mathrm{RP}-\mathrm{CrPyr}_{0}$ & $\mathrm{RP}^{-\mathrm{CrPyr}}{ }_{140}$ & $\mathrm{RP}-\mathrm{CrPyr}_{0}$ & & Transport & RP-CrPyr & $\begin{array}{l}\text { Transport } \\
\times \\
\text { RP-CrPyr }\end{array}$ \\
\hline Shannon & 5.42 & 5.15 & 5.02 & 5.33 & 0.079 & 0.520 & 0.918 & 0.102 \\
\hline Simpson & 0.01 & 0.02 & 0.02 & 0.01 & 0.002 & 0.805 & 0.599 & 0.223 \\
\hline Chaol & 1274.40 & 1215.06 & 1155.33 & 1200.78 & 23.970 & 0.202 & 0.888 & 0.306 \\
\hline ACE & 1269.03 & 1194.46 & 1140.30 & 1191.49 & 23.415 & 0.197 & 0.809 & 0.216 \\
\hline
\end{tabular}

a A $2 \times 2$ factorial arrangement with 2 supplemental levels of rumen-protected creatine pyruvate (RP-CrPyr)r in basal diets $(0$ or $140 \mathrm{~g} / \mathrm{d}$ for each cattle) and 2 transport treatment (transport 0 or $12 \mathrm{~h}$ before slaughter)

b Transport $\times$ RP-CrPyr, the interaction between Transport and RP-CrPyr

Based on the principle of similarity greater than 97\%, 1723 OTUs were obtained by the Venn diagram (Fig. 1A). Group T_CrPyr140 shared 1252 OTUs with group $\mathrm{T}_{-}$ CrPyr0, and 235 and 134 OTUs were unique to group T_CrPyr140 and group T_CrPyr0, respectively (Fig. 1B).

To measure the extent of similarity between the microbial communities, beta diversity was calculated using a weighted normalized UniFrac, and PCoA was performed. As shown in Fig. 2, these microbial community profiles from the four groups were not significantly different $(P=0.068)$, but the distance between group T_CrPyr0 and group T_CrPyr140 was obviously separated, which indicated that with transport treatment, RP-CrPyr supplementation could affect the composition of the flora in rumen fluid compared with the RP-CrPy unsupplemented group.

\section{Bacterial composition}

Phylogenetic analysis identified nineteen phyla from the rumen fluid, four of which had a relative abundance $>0.3 \%$ of the community, and the most abundant phyla were Bacteroidetes, Firmicutes, Actinobacteria, and Unclassified_k_norank_d_Bacteria (Fig. 3A), and their relative abundance showed no significant difference among the four groups. With transport treatment, group T_CrPyr140 decreased Bacteroidetes abundance and increased Firmicutes abundance compared with group T_CrPyrO (Fig. 4B, $P<0.05$ ). 

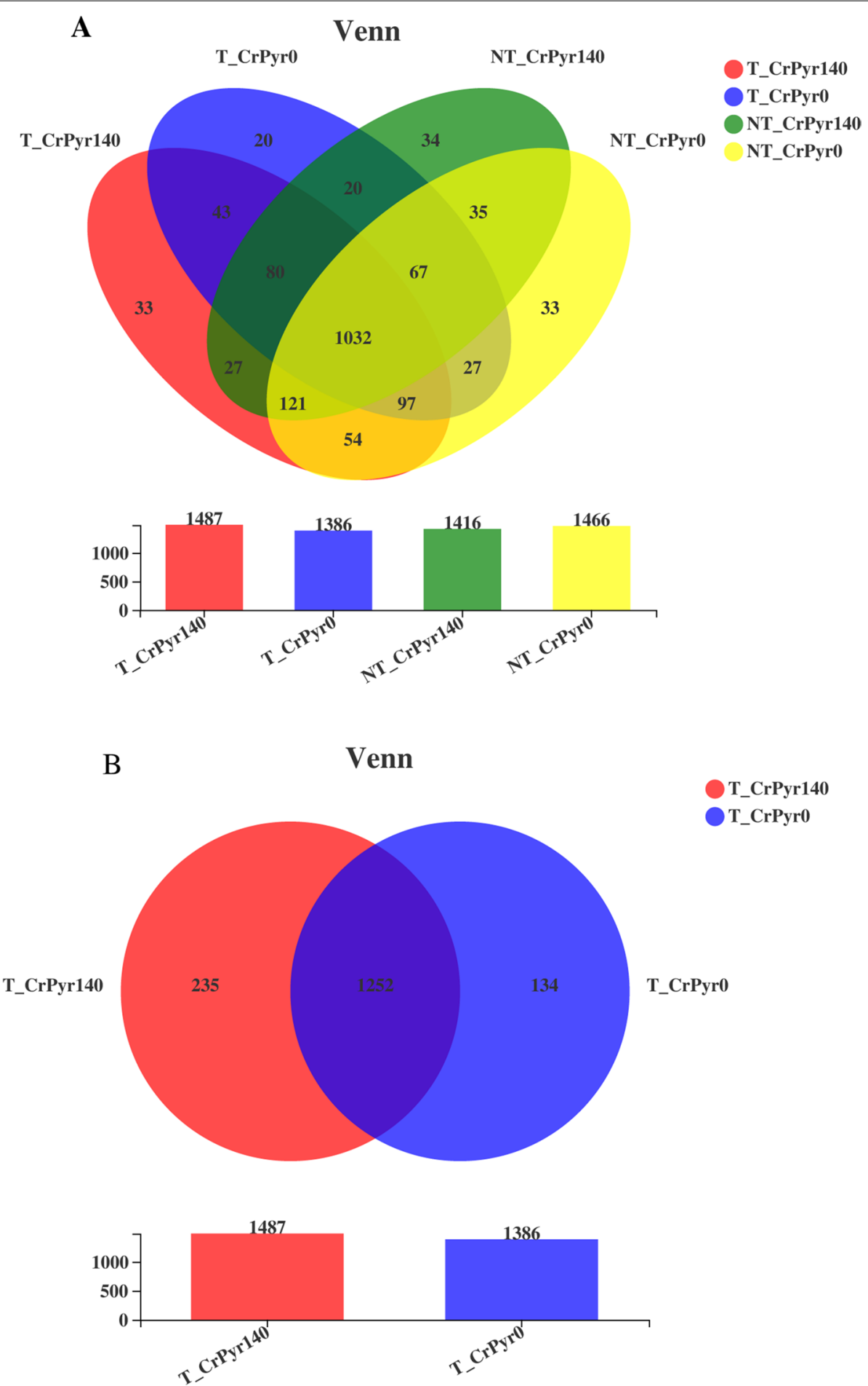

Fig. 1 Operational taxonomic unit (OTU) Venn diagram among different groups. T_CrPyr140:Transport treatment + 140 g/d/cattle rumen-protected creatine pyruvate; T_CrPyr0:Transport treatment $+0 \mathrm{~g} / \mathrm{d} /$ cattle rumen-protected creatine pyruvate. NT_CrPyr140: Nontransport treatment $+140 \mathrm{~g} / \mathrm{d} /$ cattle rumen-protected creatine pyruvate; NT_CrPyrO: Nontransport treatment $+140 \mathrm{~g} / \mathrm{d} /$ cattle rumen-protected creatine pyruvate 

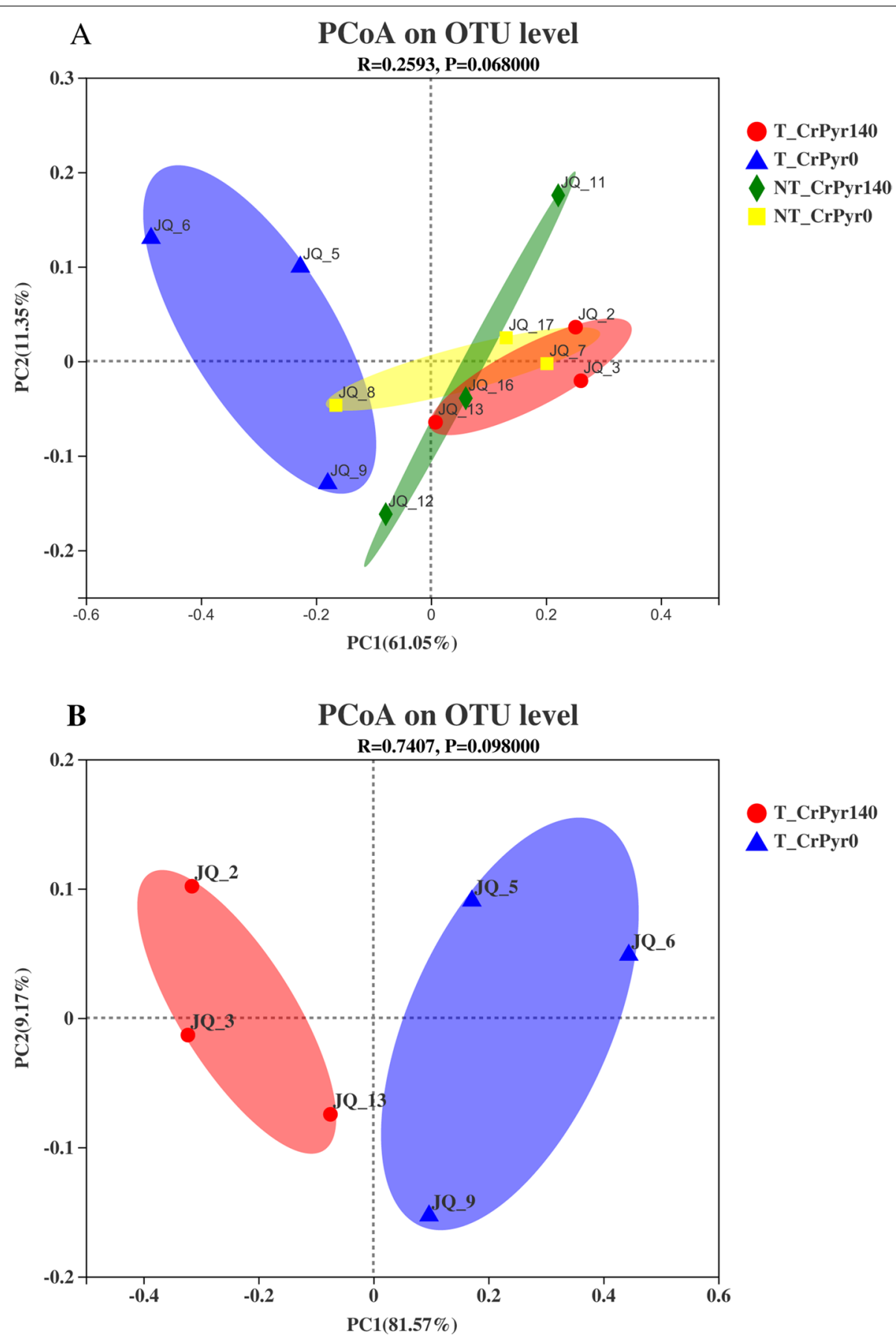

Fig. 2 Principal coordinate analysis (PCOA) plot based on OTU abundance. T_CrPyr140:Transport treatment + 140 g/d/cattle rumen-protected creatine pyruvate; T_CrPyr0:Transport treatment $+0 \mathrm{~g} / \mathrm{d} /$ cattle rumen-protected creatine pyruvate. NT_CrPyr140: Nontransport treatment $+140 \mathrm{~g} / \mathrm{d}$ /cattle rumen-protected creatine pyruvate; NT_CrPyr0: Nontransport treatment $+140 \mathrm{~g} / \mathrm{d} /$ cattle rumen-protected creatine pyruvate

At the genus level, 247 genera were shown in the ruminal fluid, thirteen of which had a relative abundance greater than $0.3 \%$ of the community, including Prevotella, Rikenellaceae_RC9_gut_group,
norank_f_Muribaculaceae, Succiniclasticum, norank $f_{-}$ UCG_-011, norank_f_F082, Lachnospiraceae_ NK3A20_group, NK4A214_group, Ruminococcus, Christensenellaceae_R-7_group, CAG-352, UCG004, and unclassified_k_norank_d_Bacteria (Fig. 3B). 


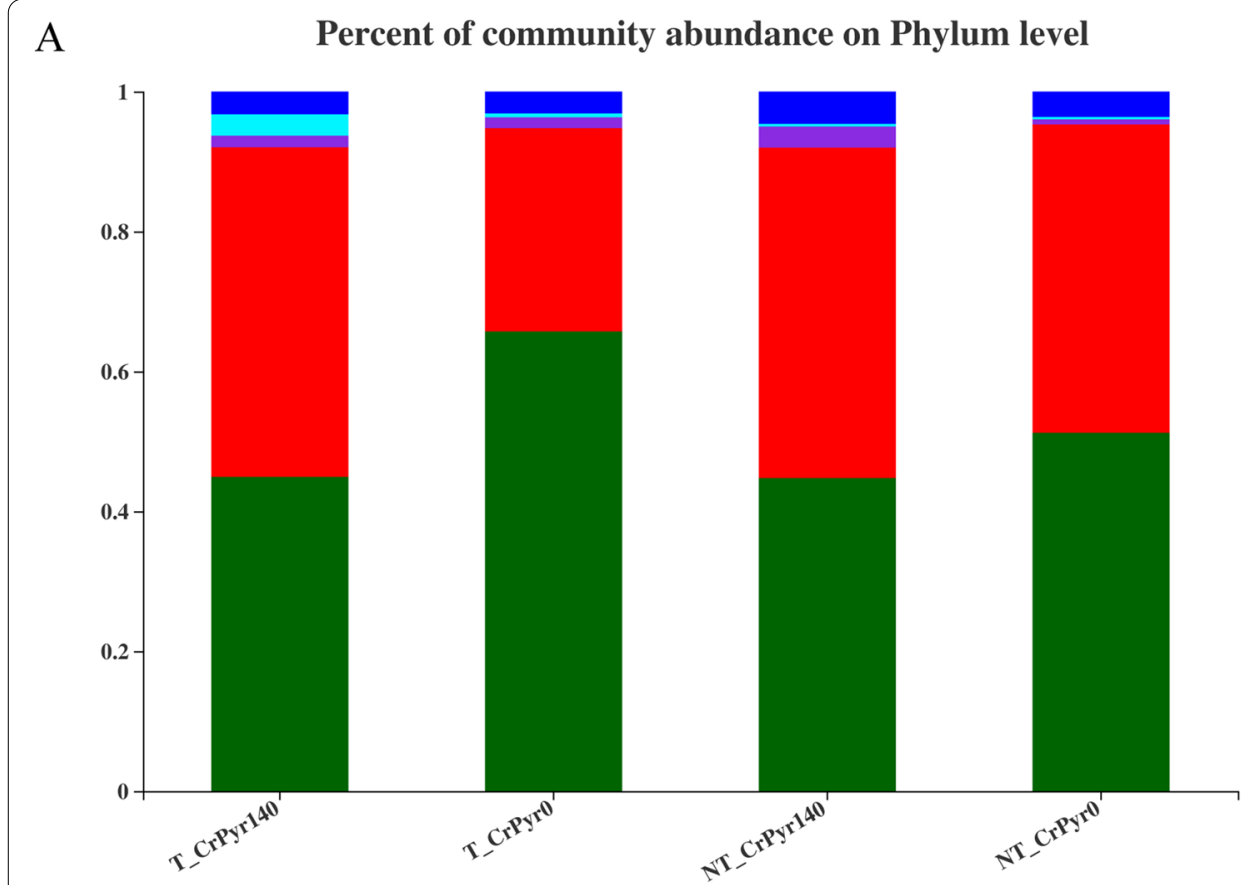

B Percent of community abundance on Genus level

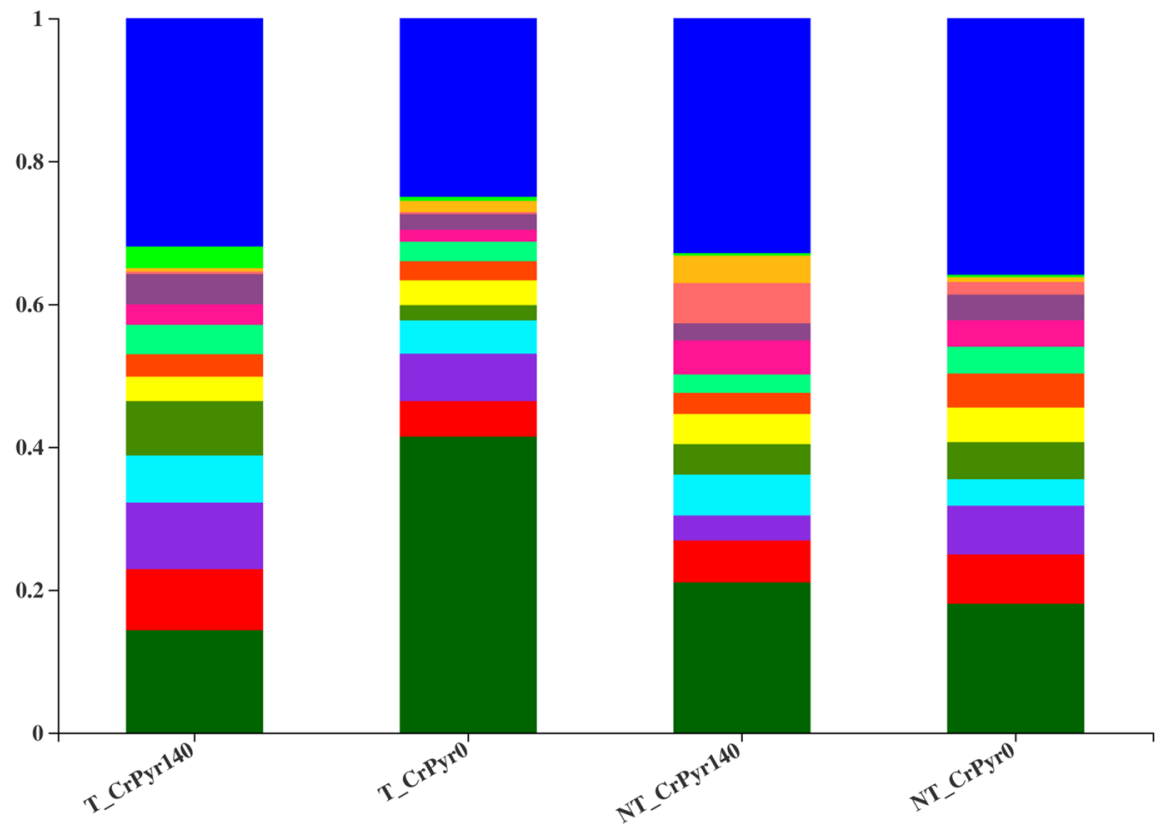

Prevotella
Rikenellaceae_RC9_gut_group
norank_f_Muribaculaceae
Succiniclasticum
norank_f_UCG-011
norank_f_F082
Lachnospiraceae_NK3A20_group
NK4A214_group
Ruminococcus
Christensenellaceae_R-7_group
CAG-352
UCG-004
unclassified_k_norank_d_Bacteria
others

Fig. 3 Bar plots showing the average relative abundance of bacterial phyla (\%) in the rumen at the phylum level (A) and genus level (B). Data represent the abundance at greater than $0.03 \%$ of the community among these four groups. T_CrPyr140: Transport treatment $+140 \mathrm{~g} / \mathrm{d} / \mathrm{cattle}$ rumen-protected creatine pyruvate; T_CrPyr0: Transport treatment + $0 \mathrm{~g} / \mathrm{d} /$ cattle rumen-protected creatine pyruvate. NT_CrPyr140: Nontransport treatment $+140 \mathrm{~g} / \mathrm{d} /$ cattle rumen-protected creatine pyruvate; NT_CrPyr0: Nontransport treatment + $140 \mathrm{~g} / \mathrm{d} /$ cattle rumen-protected creatine pyruvate 
Among them, group T_CrPyr0 increased the ruminal Prevotella abundance of beef cattle compared with group NT_CrPyr0 (Fig. 4C, $P<0.05$ ), while group $\mathrm{T}_{-}$ CrPyr140 decreased the Prevotella abundance compared with group T_CrPyr0 (Fig. 4D, $P<0.05$ ).

\section{Correlation between rumen microbiota and physiological variables}

The relationship between ruminal microbiota abundance (top 5 species) and physiological parameters was used to analyse the evaluated correlations. At the phylum level (Fig. 5A), serum LPS was positively correlated with Bacteroidetes $(P<0.05)$. The rumen fermentation characteristics of ruminal acetate were negatively correlated with Actinobacteriota $(P<0.05)$. For the immune system, serum TNF- $\alpha$ was positively correlated with Actinobacteriota. The abundance of unclassified_k_norank_d_bacteria was negatively correlated with serum IL-6. At the genus level (Fig. 5B), the abundance of Prevotella was negatively correlated with ruminal $\mathrm{pH}$ but positively correlated with ruminal MCP $(P<0.05)$. The rumen fermentation characteristics of acetate and TVFA were negatively correlated with norank $f_{-}$Muribaculaceae $(P<0.05)$. IgG was positively correlated with norank $f_{-}$ UCG-011 $(P<0.05)$.

\section{Discussion}

During long-distance transportation, beef cattle encounter continuous and strong stimuli such as driving, loading and unloading, vibration, crowding, noise, temperature and humidity changes, water and food restriction, fatigue, etc. In response to transport stress, the hypothalamicpituitary-adrenal (HPA) axis and/or sympathetic nervous system (SNS) were activated, which in turn regulated the corresponding hormones secreted by the endocrine glands. Of these, cortisol is often elevated in the stress reaction of cattle. Elevated cortisol concentrations can promote the metabolism of carbohydrates, fat, and protein, provide energy for the body, and ultimately improve the adaptability of cattle to stress [12]. However, excessive cortisol can accelerate protein decomposition and inhibit protein synthesis, resulting in weight loss. It has been reported in beef cattle that cortisol was increased on day 1 but not $6 \mathrm{~h}$ after transportation (travel time, $14 \mathrm{~h}$; distance, $1000 \mathrm{~km}$; speed, $60-70 \mathrm{~km} / \mathrm{h}$ ) [4], perhaps suggesting that the cortisol level gradually increased after transportation. In this study, there was no significant elevation of cortisol in response to transport treatment, which may be because cortisol was measured $2 \mathrm{~h}$ after transportation. Creatine monohydrate was found to be a cortisol blocker; when combined with pyruvate, pyruvylcreatine is a useful composition comprised of pyruvate and a cortisol blocker [13]. Correspondingly, the present study showed that diet supplemented with RP-CrPyr decreased the serum cortisol concentration of beef cattle, which means that RP-CrPyr could alleviate the transport stress of beef cattle.

During transportation, the imbalance between free radical production and antioxidant mechanisms induces oxidative stress and damage to the body [14]. MDA is the final product of lipid peroxidation and is utilized as an indicator of oxidative stress [15]. T-AOC is composed of antioxidants and antioxidant enzymes and is an important evaluation index that reflects oxidative damage in the body [16]. SOD, GSH-Px, and CAT are major antioxidant enzymes. SOD can scavenge superoxide radicals and convert them into hydrogen peroxide $\left(\mathrm{H}_{2} \mathrm{O}_{2}\right)$, lipid hydroperoxides are reduced to the corresponding alcohols by GSH-Px, and CAT can decompose $\mathrm{H}_{2} \mathrm{O}_{2}$ into harmless water $[17,18]$. As reported previously, transport stress may cause oxidative stress in the brains of chicks by increasing the production of lipid peroxidation and free radicals and reducing the activities of antioxidant enzymes and glutathione [19]. On the other hand, dietary supplementation with creatine reduced the concentration of MDA and increased the activity of SOD in Yili horses [20]. In the current study, transport treatment significantly decreased the serum CAT activity and T-AOC, which means that transportation results in oxidative damage to beef cattle. While dietary supplementation with RP-CrPyr tended to decrease the amount of serum MDA, the result indicates that RP-CrPyr could reduce oxidative stress to some extent. This may be because creatine and pyruvate are both energetic and antioxidant substances [21], and RP-CrPyr can be absorbed in the small intestine in the form of creatine and pyruvate in beef cattle.

TSSBC is reported to be associated with alterations in immune function induced by transport stress and increased exposure to pathogens [22]. In the present study, transport treatment significantly decreased the serum IgA level of beef cattle. It is well known that IgA can eliminate a large number of antigens in a noninflammatory form, which is a very beneficial immune

\footnotetext{
(See figure on next page.)

Fig. 4 Analysis of species composition difference. At the phylum level, group T_CrPyr0 vs group NT_CrPyrO (A) and group T_CrPyr140 vs group T_CrPyrO. At the genus level, compare between T_CrPyr0 and NT_CrPyrO (C), T_CrPyr140 and T_CrPyr0 (D).T_CrPyr140: Transport treatment $+140 \mathrm{~g} / \mathrm{d} /$ cattle rumen-protected creatine pyruvate; T_CrPyrO:Transport treatment $+0 \mathrm{~g} / \mathrm{d} /$ cattle rumen-protected creatine pyruvate. NT_ CrPyr140: Nontransport treatment + $140 \mathrm{~g} / \mathrm{d} / \mathrm{cattle}$ rumen-protected creatine pyruvate; NT_CrPyrO: Nontransport treatment + $140 \mathrm{~g} / \mathrm{d} / \mathrm{cattle}$ rumen-protected creatine pyruvate
} 
A Student's t-test bar plot on Phylum level

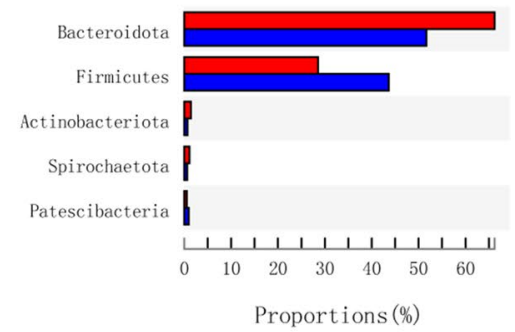

95\% confidence intervals

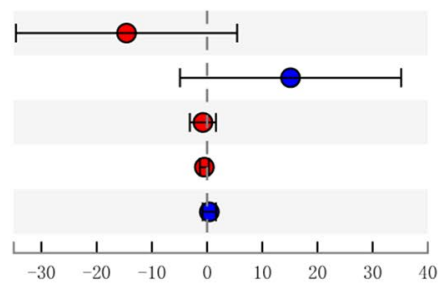

Difference between proportions $(\%)$
T_CrPyr0

- NT_CrPyr0

0.1133

0. 1041

0.4353

0.174

0.391

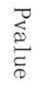

$\square$ T_CrPyr140 $\square \mathrm{T} \_$CrPyr0

95\% confidence intervals

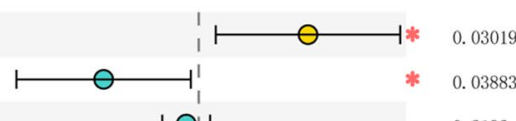

0.2192

0.7785

0.1505

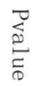
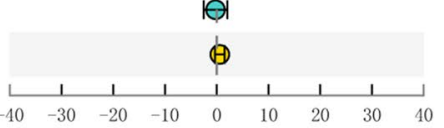

Difference between proportions $(\%)$

Proportions (\%)

95\% confidence intervals
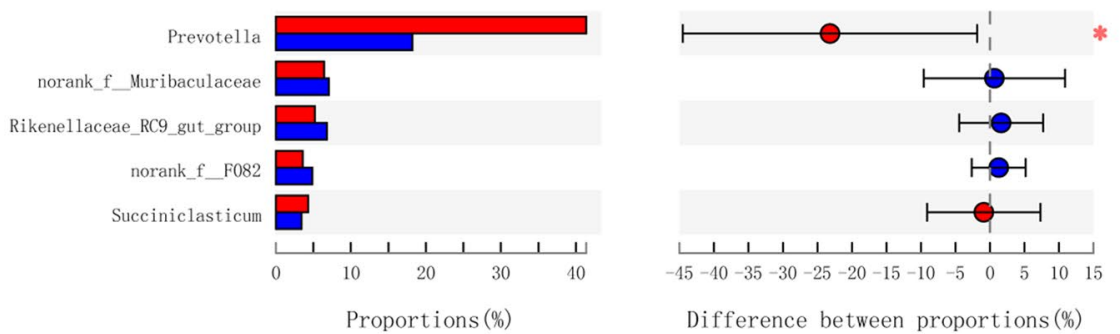

0.03928

0.87

0.4994

0.4165

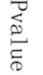

0.7794

D Student' s t-test bar plot on Genus level

95\% confidence intervals
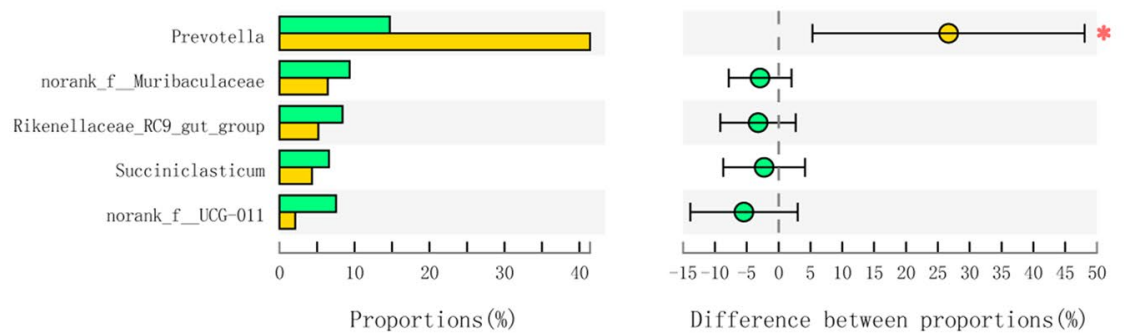

0.02561

0.1753

0.2053

0.3797

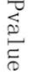

0.1476

Difference between proportions $(\%)$

Fig. 4 (See legend on previous page.) 


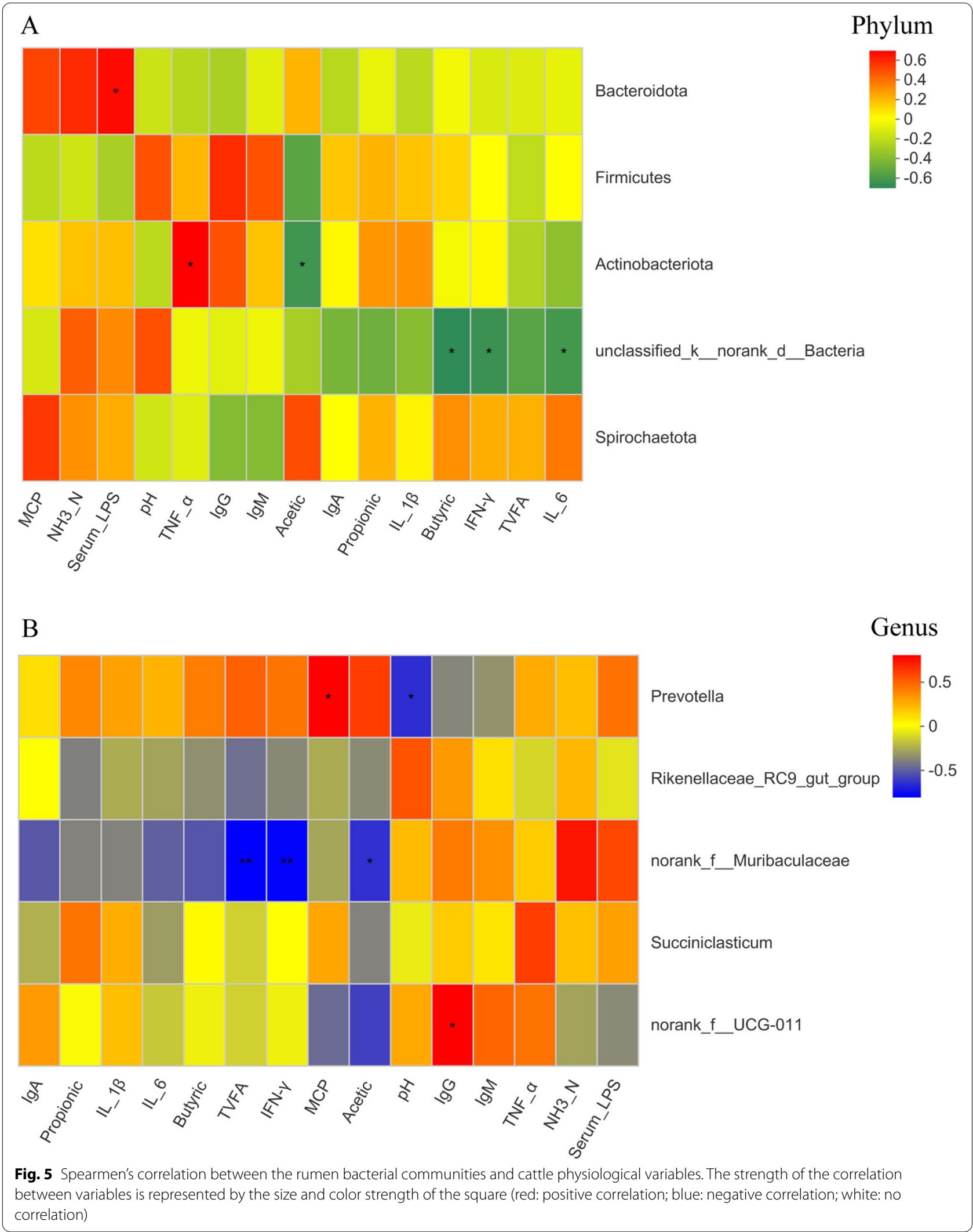


effect for maintaining the internal environment of the body. Moreover, IgA traverses the gut wall from blood into the gut lumen [23]. In mucosal surfaces, IgA binds microbial antigens and influences bacterial gene expression to regulate microbiota growth and epithelial attachment, thereby mediating microbial homeostasis [24]. The reduction in serum IgA levels caused by transport stress in this study might contribute to the decreased immune function and disordered microflora. Correspondingly, group T_CrPyr0 numerically increased rumen Bacteroidota abundance and decreased Firmicutes abundance and significantly increased the Prevotella abundance of beef cattle compared with group NT_CrPyr0. Bacteroidota and Prevotella are gram-negative bacteria, and LPS is closely bound to the surface of gram-negative bacteria and is released when the bacteria grow rapidly or die [25]. Therefore, the dysbiosis of gram-negative bacteria in the rumen microbiome of beef cattle in group $T_{-}$CrPyr0 contributed to the elevated levels of rumen and serum LPS. In contrast, in the present study, compared with group T_CrPyr0, group T_CrPyr140 significantly decreased the abundance of rumen Bacteroidota and Prevotella and increased Firmicutes abundance, while dietary supplementation with RP-CrPyr decreased the level of serum LPS.

The inflammatory response initiated by LPS could induce the expression of inflammatory cytokines (such as IL-1 $\beta$, Il-6, IFN- $\gamma$, TNF- $\alpha$ ) through a series of signal transduction pathways $[26,27]$. Previous research found that the contents of TNF- $\alpha$, IFN- $\gamma$, IL- $1 \beta$, and IL- 6 were higher posttransportation than pretransportation [5]. Our data showed that transport treatment decreased the levels of serum IFN- $\gamma$, IL-1 $\beta$, and IL- 6 in beef cattle compared with those in nontransport treatment. One reason for this discrepancy in our findings may be that different transport times have different effects on inflammatory cytokines. In addition, it has also been reported that the changes in immune function and cytokines during transportation were not simply rising and falling but a dynamic process [28]. Recently, Zhu et al. [29] identified that extracellular DNA (eDNA) was a nutritional trigger of Mycoplasma bovis (M. bovis, the main pathogenic microorganism of TSSBC) cytotoxicity, and the release of massive amounts of prokaryotic DNA induced by bacteriophages and antimicrobial drugs was noteworthy. A review showed that increased glucocorticoid release could reduce the diversity and richness of intestinal microorganisms (reviewed by Sherwin et al. [30]); therefore, transport stress may increase the release of eDNA from rumen microbiota and contribute to $M$. bovis proliferation. However, in the present study, there was no significant effect of the transport of RP-CrPyr on the contents of rumen eDNA and serum eDNA.
Rumen fermentation parameters $\left(\mathrm{pH}, \mathrm{NH}_{3}-\mathrm{N}, \mathrm{MCP}\right.$, VFA) are important indicators to evaluate rumen health. Previous studies reported that transportation decreased the rumen fluid $\mathrm{pH}$ value $[4,5]$, but we found that transport treatment tended to increase the $\mathrm{pH}$ value. This was because of the interaction of transport and RP-CrPyr on $\mathrm{pH}$. When comparing group T_CrPyrO individually to group NT_CrPyro, the $\mathrm{pH}$ value was numerically lower in group T_CrPyr0, while group T_CrPyr140 significantly increased the $\mathrm{pH}$ value. Rumen $\mathrm{NH}_{3}-\mathrm{N}$ concentration can be influenced by dietary protein breakdown, $\mathrm{NH}_{3}$ utilization by microbes, absorption by the rumen wall, and urea hydrolysis in the rumen. Our results showed that transport treatment significantly increased the concentration of $\mathrm{NH}_{3}-\mathrm{N}$, which may be attributable to the abundance of proteolytic bacteria being increased under transport stress, such as Prevotella, which promotes the decomposition of protein in feed and produces ammonia, resulting in the increase of $\mathrm{NH}_{3}-\mathrm{N}$. Consistently, for dairy cows suffering heat stress, the concentration of rumen $\mathrm{NH}_{3}-\mathrm{N}$ was also increased [31]. VFAs, the main metabolic product of rumen fermentation, provide approximately $70 \%$ of the host's metabolic energy and have a direct relationship with the rumen microbial community [32]. Previous studies reported that transport stress has an influence on ruminal carbohydrate fermentation, but the results have been inconsistent $[4,5]$. In the present study, transport treatment significantly decreased the contents of rumen TVFAs, acetate, and butyrate, which may be because beef cattle withdrew feed during transport, thereby reducing the concentration of rumen fermentation substrate and affecting microorganism fermentation and the generation of VFAs. RP-CrPyr supplementation also reduced the amount of TVFA and butyrate compared with RPCrPyr unsupplemented groups. This may be because RPCrPyr was decomposed into pyruvate and creatine in the small intestine and absorbed, which can provide energy for the body as energy substances, therefore sparing VFA for energy supply.

One previous study reported that in dairy cattle, preweaning stress did not change the microbial community constantly in the rumen and faeces based on the analysed species richness [33]. Under heat stress, dietary supplementation with CrPyr did not influence the $\alpha$-diversity index [11]. In this study, to analyse ruminal bacterial richness and diversity indices, we found that there was no difference in the Chaol, Shannon, Simpson, ACE and OTU indices among the four groups. These results were similar to the results of a previous study. In this study, our results indicated that Bacteroidetes, Firmicutes, and Actinobacteriota were the dominant flora at the phylum level among the four groups. Additionally, previous studies reported that Bacteroidetes and 
Firmicutes were the most dominant flora of ruminal microorganisms in beef cattle [34]. The major classes within the phylum Bacteroidetes in the rumen were found to be hemicellulolytic bacteria, amylolytic bacteria and proteolytic bacteria [35, 36]. The phylum Bacteroidetes has a superb ability to utilize nutrients, including simple and complex sugars and polysaccharides, for its growth to adapt to environmental changes and stresses [37]. Increased phylum Bacteroidetes was also observed in weaning-stressed piglets [38] and heat-stressed birds $[39,40]$. The phylum Firmicutes contains a large number of cellulolytic bacteria and thus has a stronger fibre decomposition ability $[41,42]$. During heat stress, ruminal cellulolytic bacteria were downregulated, while amylolytic bacteria were upregulated in dairy cows [43]. Similarly, in the present study, transport stress numerically increased Bacteroidetes phylum (containing amylolytic bacteria) abundance and decreased Firmicutes (containing cellulolytic bacteria) abundance. Furthermore, the Prevotella genus was the predominant genus belonging to the Bacteroidetes phylum, and its level was significantly increased in beef cattle within T_CrPyrO. In contrast, transported beef cattle fed RP-CrPyr had lower levels of the Bacteroidetes phylum and Prevotella genus and higher levels of the Firmicutes phylum, which means that RP-CrPy promotes the restoration of normal rumen flora under transport stress. Recently, there has been increasing concern about the relationship between stress and gut microbiota. Depression patients had an increased plasma cortisol level [44], a higher proportion of Bacteroidetes and a lower proportion of Firmicutes [45] and upregulation of Prevotella genus abundance in the gut microbiome [46]. Therefore, transport stress caused an increase in the abundance of Bacteroidetes and Prevotella in the rumen microbial community, which may be related to increased serum cortisol levels, while RP-CrPy reduced the cortisol level of transported beef cattle, which can stimulate the restoration of the natural flora.

\section{Conclusion}

In conclusion, transport stress elicited negative changes in endocrine parameters, antioxidant status, immunity, rumen fermentation and microorganisms of beef cattle. Dietary supplementation with RP-CrPyr might be beneficial to alleviate transport stress by decreasing serum cortisol and LPS levels and promoting the restoration of the rumen natural flora.

\section{Methods}

\section{Animal treatments and experimental Diets}

Twenty male Chinese Simmental crossbred cattle (initial body weight $=659 \pm 16 \mathrm{~kg}$ ) of 18 months of age were randomly allocated to four groups using a $2 \times 2$ factorial arrangement with two RP-CrPyr supplemental levels $(0$ or $140 \mathrm{~g} / \mathrm{d})$ in basal diets and two types of transport $(5 \mathrm{~min}$ or $12 \mathrm{~h}$ ). Compared to the $12 \mathrm{~h}$ of transportation, the $5 \mathrm{~min}$ of transportation was relatively short; for ease of presentation and understanding, 5 min of transportation was described as nontransport. That were T_CrPyr140 (12 h transport $+140 \mathrm{~g} / \mathrm{d}$ RPCrPyr in basal diet); T_CrPyr0 (12 h transport $+0 \mathrm{~g} / \mathrm{d}$ RP-CrPyr in basal diet); NT_CrPyr140 (5 min transport $+140 \mathrm{~g} / \mathrm{d}$ RP-CrPyr in basal diet); NT_CrPyr0 (5 min transport $+0 \mathrm{~g} / \mathrm{d} \mathrm{RP}-\mathrm{CrPyr}$ in basal diet). After feeding for 30 days, three animals from each treatment were selected for euthanasia and to collect samples. The catthe in the nontransport group were fed at $16: 00 \mathrm{pm}$ on September 16, 2020, loaded onto trucks (at 04:00 am on September 17,2020$)$ to slaughter near the farm $(1 \mathrm{~km})$, which took approximately $5 \mathrm{~min}$. After unloading, the cattle rested for $2 \mathrm{~h}$ and were slaughter by electrical stunning and exsanguination (at 06:00 am on September 17, 2020). Briefly, the cattle in the nontransport group were fasted for approximately $14 \mathrm{~h}$ (12 h fasting prior transport, $5 \mathrm{~min}$ transport, $2 \mathrm{~h}$ lairage followed by slaughter). The transported beef cattle were fed at 06:00 am on September 16, 2020 and transported from Xuchang, Henan Province, to Nanyang, Henan Province, for $12 \mathrm{~h}$ at an average speed of $60-70 \mathrm{~km} / \mathrm{h}$. The journey started at 13:00 pm on September 16, 2020, and the cattle arrived at 01:00 am on September 17, 2020. The outside temperature was between 22 and $32^{\circ} \mathrm{C}$ with a relative humidity of $30-72 \%$. After unloading, the cattle were allowed to rest for $2 \mathrm{~h}$ and then electrical stunning and exsanguination (03:00 am on September 17, 2020). Briefly, the cattle in the transport group were fasted for approximately $21 \mathrm{~h}(7 \mathrm{~h}$ fasting prior transport, $12 \mathrm{~h}$ transport, $2 \mathrm{~h}$ lairage followed by slaughter).

Table 5 Composition and nutrient levels of experimental diet (air-dry basis, \%)

\begin{tabular}{|c|c|c|c|}
\hline Ingredients & Content & Nutrient levels & Content \\
\hline Wheat Straw & 40.00 & Dry matter & 87.20 \\
\hline Corn & 43.74 & Crude protein & 13.52 \\
\hline wheat bran & 3.66 & Crude fat & 2.47 \\
\hline Soybean meal & 9.12 & Ash & 7.86 \\
\hline Sodium bicarbonate & 0.42 & Neutral detergent fiber & 36.18 \\
\hline Premix ${ }^{a}$ & 3.06 & Acid detergent fiber & 15.81 \\
\hline Tatol & 100 & & \\
\hline
\end{tabular}

a The premix (per kg of diet) is: 80000 IU of vitamin A, 20000 IU of vitamin D3, $280 \mathrm{mg}$ of vitamin E, $4100 \mathrm{mg}$ of $\mathrm{Fe}, 1100 \mathrm{mg}$ of $\mathrm{Mn}, 800 \mathrm{mg}$ of $\mathrm{Zn}, 265 \mathrm{mg}$ of $\mathrm{Cu}$, $120 \mathrm{~g}$ of $\mathrm{Ca}$ and $35 \mathrm{~g}$ of $\mathrm{P}$ 
CrPyr (purity of 99.9\%) was purchased from Shanghai Jinli Technology Co., Ltd. (Shanghai, China). The production of RP-CrPyr was entrusted to Hangzhou King Techina Feed Co., Ltd. (Hangzhou, China), the CrPyr coating rate was $40 \%$, the rumen bypass ratio $(12 \mathrm{~h})$ was $82.8 \%$, and the dissolution of intestinal fluid $(12 \mathrm{~h})$ was $78.6 \%$. Our previous study results indicated that Jinjiang cattle fed $60 \mathrm{~g} / \mathrm{d}$ CrPyr had a better resistance to heat stress [11]. Therefore, the present experiment selected a $140 \mathrm{~g} / \mathrm{d}$ dose to explore the regulatory mechanism of RP-CrPyr in beef cattle. RP-CrPyr was added to the concentrates, divided into two daily feeds (06:00 and 16:00). After the rice straw was fed, concentrate was given. All cattle were housed in individual solid concrete floor pens in a closed cowshed, while clean, freshwater was available at all times. The composition and nutrient levels of the experimental diet are shown in Table 5.

\section{Sample collection}

Before slaughter, $15 \mathrm{~mL}$ of blood sample was taken from the jugular vein of cattle into evacuated nonanticoagulative tubes. The blood samples were centrifuged at $3000 \mathrm{~g}$ $\left(10 \mathrm{~min}, 4^{\circ} \mathrm{C}\right)$ to collect serum samples, and then stored at $-20^{\circ} \mathrm{C}$ to measure indices. Then, the beef cattle were slaughtered by electrical stunning and exsanguination. After slaughter, $400 \mathrm{~mL}$ of rumen fluid was collected from the upper, middle, and lower sites in the rumen and squeezed through four layers of gauze. The rumen fluid was immediately measured for $\mathrm{pH}$ using a portable $\mathrm{pH}$ metre (HANNA Instruments, Cluj-Napoca, Romania). Then, the samples were divided into three portions. The first $8 \mathrm{~mL}$ of rumen fluid was mixed with $2 \mathrm{~mL}$ of $25 \%$ (wt/vol) metaphosphoric acid and used for VFA analysis. One subsample $(10 \mathrm{~mL})$ of rumen fluid was mixed with $2 \mathrm{~mL}$ of $\mathrm{H}_{2} \mathrm{SO}_{4}(1 \% \mathrm{vol} / \mathrm{vol})$ for determination of ammonia nitrogen $\left(\mathrm{NH}_{3}-\mathrm{N}\right)$, and another subsample $(10 \mathrm{~mL})$ of rumen fluid was used for microbial crude protein (MCP) and $16 \mathrm{~S}$ rDNA sequencing analysis. These samples were frozen in liquid nitrogen and stored at $-80^{\circ} \mathrm{C}$ until DNA extraction.

\section{Chemical analyses}

The VFA concentrations in the rumen fluid samples were determined using gas chromatography (Shimadzu GC-2014, Japan) equipped with a capillary column (Stabilwax, Restek, Bellefonte, PA, USA). The $\mathrm{NH}_{3}-\mathrm{N}$ concentration was measured using a TU-1901 spectrophotometer (Beijing Purkinje General Instrument Co. Ltd., China) according to a method described by Broderick and Kang [47]. MCP production was determined according to the method of Coomassie Brilliant Blue [48].
Serum cortisol, triiodothyronine (T3), thyroxine (T4), IgG, IgA, IgM, tumour necrosis factor- $\alpha$ (TNF- $\alpha$ ), interferon $\gamma$ (IFN- $\gamma$ ), interleukin-1 (IL-1 $\beta$ ), IL-6, lipopolysaccharide (LPS) and rumen fluid LPS were determined using commercial kits (Nanjing Jiancheng Bioengineering Institute, Nanjing, China). The level of malondialdehyde (MDA), the activities of superoxide dismutase (SOD), glutathione peroxidase (GSH-Px), catalase (CAT) and total antioxidant capacity (T-AOC) in the serum samples were measured using commercial kits (Nanjing Jiancheng Bioengineering Institute). Serum and rumen fluid eDNA content analysis was assessed by a dsDNA HS Assay Kit for Qubit ${ }^{\circledR}$ (Kit: Shanghai Yisheng Bioengineering Institute, Shanghai, China).

\section{S rDNA sequencing analysis}

According to manufacturer, microbial community genomic DNA was extracted from ruminal fluid samples using the E.Z.N.A. ${ }^{\circledR}$ soil DNA Kit (Omega Bio-tek, Norcross, GA, U.S.). The DNA extract was checked on $1 \%$ agarose gel, and DNA concentration and purity were determined with NanoDrop 2000 UV-vis spectrophotometer (Thermo Scientific, Wilmington, USA). The hypervariable region V3-V4 of the bacterial 16S rRNA gene was amplified with primer pairs $338 \mathrm{~F}\left(5^{\prime}\right.$-ACTCCT ACGGGAGGCAGCAG-3 ${ }^{\prime}$ ) and 806R (5'-GGACTA CHVGGGTWTCTAAT-3') by an ABI GeneAmp ${ }^{\circledR} 9700$ PCR thermocycler (ABI, CA, USA). The PCR amplification of $16 \mathrm{~S}$ rRNA gene was performed as follows: initial denaturation at $95^{\circ} \mathrm{C}$ for $3 \mathrm{~min}$, followed by 27 cycles of denaturing at $95^{\circ} \mathrm{C}$ for $30 \mathrm{~s}$, annealing at $55^{\circ} \mathrm{C}$ for $30 \mathrm{~s}$ and extension at $72^{\circ} \mathrm{C}$ for $45 \mathrm{~s}$, and single extension at $72^{\circ} \mathrm{C}$ for $10 \mathrm{~min}$, and end at $4^{\circ} \mathrm{C}$. The PCR mixtures contain $5 \times$ TransStart FastPfu buffer $4 \mu \mathrm{L}, 2.5 \mathrm{mM}$ dNTPs $2 \mu \mathrm{L}$, forward primer $(5 \mu \mathrm{M}) 0.8 \mu \mathrm{L}$, reverse primer $(5 \mu \mathrm{M})$ $0.8 \mu \mathrm{L}$, TransStart FastPfu DNA Polymerase $0.4 \mu \mathrm{L}$, template DNA $10 \mathrm{ng}$, and finally $\mathrm{ddH}_{2} \mathrm{O}$ up to $20 \mu \mathrm{L}$. PCR reactions were performed in triplicate. The PCR product was extracted from $2 \%$ agarose gel and purified using the AxyPrep DNA Gel Extraction Kit (Axygen Biosciences, Union City, CA, USA) according to manufacturer's instructions and quantified using Quantus ${ }^{\mathrm{TM}}$ Fluorometer (Promega, USA).

Purified amplicons were pooled in equimolar and paired-end sequenced $(2 \times 300)$ on an Illumina MiSeq platform (Illumina, San Diego, USA) according to the standard protocols by Majorbio Bio-Pharm Technology Co. Ltd. (Shanghai, China). The raw reads were deposited into the NCBI BioProject database (Accession Number: PRJNA732599).

The raw $16 \mathrm{~S}$ rDNA gene sequencing reads were demultiplexed, quality-filtered by Trimmomatic and merged by FLASH with the following criteria: (i) the $300 \mathrm{bp}$ reads 
were truncated at any site receiving an average quality score of $<20$ over a $50 \mathrm{bp}$ sliding window, and the truncated reads shorter than $50 \mathrm{bp}$ were discarded, reads containing ambiguous characters were also discarded; (ii) only overlapping sequences longer than $10 \mathrm{bp}$ were assembled according to their overlapped sequence. The maximum mismatch ratio of overlap region is 0.2. Reads that could not be assembled were discarded; (iii) Samples were distinguished according to the barcode and primers, and the sequence direction was adjusted, exact barcode matching, 2 nucleotide mismatch in primer matching.

Operational taxonomic units (OTUs) with 97\% similarity cutoff were clustered using UPARSE (version 7.1, http://drive5.com/uparse/), and chimeric sequences were identified and removed. The taxonomy of each OTU representative sequence was analyzed by RDP Classifier (http://rdp.cme.msu.edu/) against the 16S rRNA database (eg. Silva 132/16s_bacteria) using confidence threshold of 0.7 .

\section{Statistical analysis}

The data were analysed with SPSS (version 17.0, IBM, Armonk, NY, USA). The four treatment groups were analysed as a $2 \times 2$ factorial arrangement using a general linear model (GLM) to elucidate the main effects of transport, RP-CyPry, and their interactions. The results are shown as the mean and standard error mean (SEM). When the interaction was significant, differences among means were determined using Tukey's multiple range test for individual comparisons. A $P$ value $<0.05$ was considered statistically significant.

\begin{abstract}
Abbreviations
CAT: Catalase; eDNA: Extracellular DNA; GSH-Px: Glutathione peroxidase; HPA:

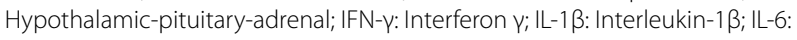
Interleukin-6; LPS: Lipopolysaccharide; MCP: Microbial crude protein; MDA: Malondialdehyde; $\mathrm{NH}_{3}-\mathrm{N}$ : Ammonia nitrogen; OTUs: Operational taxonomic units; RP-CrPyr: Rumen-protected creatine pyruvate; SEM: Standard error of the mean; SNS: Sympathetic nervous system; T3: Triiodothyronine; T4: Thyroxine; TSSBC: Transport stress syndrome of beef cattle; SOD: Superoxide dismutase; T-AOC: Total antioxidant capacity; TNF-a: Tumour necrosis factor-a; TVFA: Total volatile fatty acids.
\end{abstract}

\section{Acknowledgements}

The authors appreciate all the helps from our colleagues and collaborators.

\section{Authors' contributions}

KM and YL were responsible for the conception and design of the study. KM was responsible for data extraction and interpretation of the results, KM and $\mathrm{GL}$ carried out the statistical analysis. $Y L$ and $M Q$ acquired funding for the research project. $Y Z, X Z, Q Q$, and KOuyang supervised the research activity. $Y Z$ and $X Z$ were mainly responsible for drafting the manuscript. $K M, G L, Y L, Q Q$, and $\mathrm{MQ}$ were involved in revising the draft. All authors read and approved the final manuscript.

\section{Funding}

This work was supported by the National Natural Science Foundation of China (No. 32160814), the Youth Science Fund of Jiangxi Province
(20202BAB215010), China Agriculture Research System of MOF and MARA (CARS-37).

\section{Availability of data and materials}

The datasets analysed during the current study are available from the corresponding author on reasonable request.

\section{Declarations}

\section{Ethics approval and consent to participate}

This experiment was approved by the Committee for the Care and Use of Experimental Animals at Jiangxi Agricultural University (JXAULL-2021-10).

\section{Consent for publication}

Not applicable.

\section{Competing interests}

The authors declare that they have no competing interests.

\section{Author details}

1 Jiangxi Province Key Laboratory of Animal Nutrition, College of Animal Science and Technology, Jiangxi Agricultural University, Nanchang, China. ${ }^{2}$ Jiangxi Province Key Laboratory of Animal Nutrition/Animal Nutrition and Feed Safety Innovation Team, College of Animal Science and Technology, Jiangxi Agricultural University, Nanchang, China. ${ }^{3}$ College of Animal Science and Technology, Jiangxi Agricultural University, Nanchang, China.

Received: 12 November 2021 Accepted: 29 December 2021

Published online: 15 January 2022

\section{References}

1. González LA, Schwartzkopf-Genswein KS, Bryan M, Silasi R, Brown F. Relationships between transport conditions and welfare outcomes during commercial long haul transport of cattle in North America. J Anim Sci. 2012:90:3640-51.

2. Keiko JK, Pendell DL. Market impacts of reducing the prevalence of bovine respiratory disease in United States beef cattle feedlots. Front Vet Sci. 2017:4:189.

3. Thomas M, Webb M, Ghimire S, et al. Metagenomic characterization of the effect of feed additives on the gut microbiome and antibiotic resistome of feedlot cattle. Sci Rep. 2017;7:12257.

4. Deng L, He C, Zhou Y, Xu L, Xiong H. Ground transport stress affects bacteria in the rumen of beef cattle: A real-time PCR analysis. Anim Sci J. 2017:88:790-7.

5. Li F, Shah AM, Wang Z, et al. Effects of land transport stress on variations in ruminal microbe diversity and immune functions in different breeds of cattle. Animals. 2019;9:599.

6. Freestone P, Lyte M. Stress and microbial endocrinology: Prospects for ruminant nutrition. Animal. 2010:4:1248-57.

7. Jäger R, Purpura M, Shao A, Inoue T, Kreider RB. Analysis of the efficacy, safety, and regulatory status of novel forms of creatine. Amino Acids. 2011;40:1369-83.

8. Jäger R, Metzger J, Lautmann K, et al. The effects of creatine pyruvate and creatine citrate on performance during high intensity exercise. J Int Soc Sport Nutr. 2008:5:1-9.

9. Chen J. Effects of creatine pyruvate on lipid and protein metabolism in broiler chickensDoctor's thesis:: Nanjing Agricultural University; 2011.

10. Zhao MM. Regulation mechanism of in ovo feeding of creatine pyruvate on energy metabolism and muscle development of broiler chickensDoctor's thesis:: Nanjing Agricultural University; 2017.

11. Liu L, Zhang WJ, Yu HJ, Xu LJ, Qu MR, Li YJ. Improved antioxidant activity and rumen fermentation in beef cattle under heat stress by dietary supplementation with creatine pyruvate. Anim Sci J. 2020;91:e13486.

12. Dijkstra J, Ellis JL, Kebreab E, Strathe AB, Bannink A. Ruminal pH regulation and nutritional consequences of low pH. Anim Feed Sci Technol. 2012;172:22-33. 
13. Beale PK. Composition of pyruvate and anti-cortisol compounds and method for increasing protein concentration in a mamma. U.S. Patent No. 5,756,469. Washington, DC: U.S. Patent and Trademark Office; 1998.

14. Silva WD, Machado AS, Souza MA, Mello-Ca Rpes PB, Ca Rpes FP. Effect of green tea extract supplementation on exercise-induced delayed onset muscle soreness and muscular damage. Physiol Behav. 2018;194:77-82.

15. Kermanshahi $\mathrm{H}$, Moghaddam HN. Effects of supplemental vitamin $\mathrm{C}$ and chromium on metabolic and hormonal responses, antioxidant status, and tonic immobility reactions of transported broiler chickens. Biol Trace Elem Res. 2014;157:224-33.

16. Feng C, Bai K, Wang A, Ge X, Zhao Y, Zhang L, et al. Effects of dimethylglycine sodium salt supplementation on growth performance, hepatic antioxidant capacity, and mitochondria-related gene expression in weanling piglets born with low birth weight1. J Anim Sci. 2018;96(9):3791-803.

17. Meghana K, Sanjeev G, Ramesh B. Curcumin prevents streptozotocininduced islet damage by scavenging free radicals: a prophylactic and protective role. Eur J Pharmacol. 2007;577:183-91.

18. Yousef MI, Saad AA, El-Shennawy LK. Protective effect of grape seed proanthocyanidin extract against oxidative stress induced by cisplatin in rats. Food Chem Toxicol. 2009:47:1176-83.

19. Ge J, Li H, Sun F. Transport stress-induced cerebrum oxidative stress is not mitigated by activating the Nrf2 antioxidant defense response in newly hatched chicks. J Anim Sci. 2017;95:2871-8.

20. Li XB, Ma J, Nie BB, Yang JT, Qiao CJ, Yang KL. The effect of supplement with Branched-chain Amino acid, creatine on blood biochemical index of Yili horse. Xinjiang Agricult Sci. 2015;52:325-35.

21. Rieger E, Franceschi ID, Preissler T, Wannmacher C. Neuroprotective Effect of creatine and pyruvate on enzyme activities of phosphoryl transfer network and oxidative stress alterations caused by leucine administration in Wistar Rats. Neurotox Res. 2017;32:575-84.

22. Buckham Sporer KR, Burton JL, Earley B, Crowe MA. Transportation stress in young bulls alters expression of neutrophil genes important for the regulation of apoptosis, tissue remodeling, margination, and anti-bacterial function. Vet Immunol Immunopathol. 2007;118:19-29.

23. Sompayrac L. How the Immune System Works. 3rd ed. Oxford: Blackwell Publishing; 2008.

24. Huus KE, Petersen C, Finlay BB. Diversity and dynamism of IgA-microbiota interactions. Nat Rev Immunol. 2021;21:514-25.

25. Zhang R. Omics-Based approaches to assess the effects of subacute ruminal acidosis on rumen microbiota, metabolism and epithelial function in dairy cowsDoctor's thesis:: Nanjing Agricultural University; 2015.

26. Abu-Rish EY, Mansour AT, Mansour HT, Dahabiyeh LA, Aleidi SM, Bustanji Y. Pregabalin inhibits in vivo and in vitro cytokine secretion and attenuates spleen inflammation in Lipopolysaccharide/Concanavalin A -induced murine models of inflammation. Sci Rep. 2020;10:4007.

27. Mogensen TH. Pathogen recognition and inflammatory signaling in innate immune defenses. Clin Microbiol Rev. 2009;22:240-73.

28. Wang L, Wang D, Cheng Z. Effects of transportation stress on blood physiological and biochemical indexes of Angus cattle. Sci Technol. 2020;56:35-9.

29. Zhu XF, Dordet-Frisoni E, Gillard L, Marie-Claude A, Eveline H. ExtracelIular DNA: a nutritional trigger of Mycoplasma bovis cytotoxicity. Front Microbiol. 2019:10:2753.

30. Sherwin E, Bordenstein SR, Quinn JL, Dinan TG, Cryan JF. Microbiota and the social brain. Science. 2019;366:eaar2016.

31. Nasrollahi SM, Zali A, Ghorbani GR, Khani M, Maktabi H, Beauchemin KA. Effects of increasing diet fermentability on intake, digestion, rumen fermentation, blood metabolites and milk production of heat-stressed dairy cows. Animal. 2019;13:2527-35.

32. Krehbiel CR. Applied nutrition of ruminants: Fermentation and digestive physiology. Prof Anim Sci. 2014;30:129-39.

33. Meale SJ, Li SC, Azevedo P, et al. Weaning age influences the severity of gastrointestinal microbiome shifts in dairy calves. Sci Rep. 2017;7:198.

34. Thoetkiattikul H, Mhuantong W, Laothanachareon T, et al. Comparative analysis of microbial profiles in cow rumen fed with different dietary fiber by tagged 16s 16S rRNA gene pyrosequencing. Curr Microbiol. 2013;67:130-7

35. Evans NJ, Brown JM, Murray RD, et al. Characterization of novel bovine gastrointestinal tract Treponema isolates and comparison with bovine digital dermatitis treponemes. Appl Environ Microbiol. 2011;77:138-47.
36. Marais JP Therion J Mackie RI, Kistner A, Dennison C. Effect of nitrate and its reduction products on the growth and activity of the rumen microbial population. Br J Nutr. 1988;59:301-13.

37. Wexler HM. Bacteroides: the good, the bad, and the nitty-gritty. Clin Microbiol Rev. 2007;20:593-621.

38. Jiang X, Lu N, Zhao H, Yuan H, Xia D, Lei H. The Microbiome-Metabolome Response in the Colon of Piglets Under the Status of Weaning Stress. Front Microbiol. 2020;11:2055

39. Shi $D$, Bai $L, Q u Q$, et al. Impact of gut microbiota structure in heatstressed broilers. Poult Sci. 2019;98:2405-13.

40. Zhu L, Liao R, Wu N, Zhu G, Yang C. Heat stress mediates changes in fecal microbiome and functional pathways of laying hens. Appl Microbiol Biotechnol. 2019;103:461-72.

41. Turnbaugh PJ, Ley RE, Mahowald MA, Magrini V, Mardis ER, Gordon JI. An obesity-associated gut microbiome with increased capacity for energy harvest. Nature. 2006;444:1027-31.

42. Tremaroli V, Bäckhed F. Functional interactions between the gut microbiota and host metabolism. Nature. 2012:489:242-9.

43. Uyeno Y, Sekiguchi Y, Tajima K, Takenaka A, Kurihara M, Kamagata Y. An rRNA-based analysis for evaluating the effect of heat stress on the rumen microbial composition of Holstein heifers. Anaerobe. 2010;16:27-33.

44. Kelly JR, Kennedy PJ, Cryan JF, Dinan TG, Clarke G, Hyland NP. Breaking down the barriers: the gut microbiome, intestinal permeability and stress-related psychiatric disorders. Front Cell Neurosci. 2015;9:392.

45. Jiang H, Ling Z, Zhang Y, et al. Altered fecal microbiota composition in patients with major depressive disorder. Brain Behav Immun. 2015:48:186-94.

46. Lin P, Ding B, Feng C, et al. Prevotella and Klebsiella proportions in fecal microbial communities are potential characteristic parameters for patients with major depressive disorder. J Affect Disord. 2017;207:300-4.

47. Broderick GA, Kang JH. Automated simultaneous determination of ammonia and total amino acids in ruminal fluid and in vitro media. J Dairy Sci. 1980;63:64-75.

48. Zinn RA, Owens FN. A rapid procedure for purine measurement and its use for estimating net ruminal protein synthesis. Can J Anim Sci. 1986:66:157-66.

\section{Publisher's Note}

Springer Nature remains neutral with regard to jurisdictional claims in published maps and institutional affiliations.

Ready to submit your research? Choose BMC and benefit from

- fast, convenient online submission

- thorough peer review by experienced researchers in your field

- rapid publication on acceptance

- support for research data, including large and complex data types

- gold Open Access which fosters wider collaboration and increased citations

- maximum visibility for your research: over 100M website views per year

At BMC, research is always in progress.

Learn more biomedcentral.com/submissions 